\title{
Strong Local Non-Determinism of Sub-Fractional Brownian Motion
}

\author{
Nana Luan \\ School of Insurance and Economics, University of International Business and Economics, Beijing, China \\ Email: luannana@uibe.edu.cn
}

Received 30 August 2015; accepted 27 November 2015; published 30 November 2015

Copyright (C) 2015 by author and Scientific Research Publishing Inc.

This work is licensed under the Creative Commons Attribution International License (CC BY). http://creativecommons.org/licenses/by/4.0/

(c) () Open Access

\section{Abstract \\ Let $X^{H}=\left\{X^{H}(t), t \in \mathbb{R}_{+}\right\}$be a subfractional Brownian motion in $\mathbb{R}^{d}$. We prove that $X^{H}$ is strongly locally nondeterministic. \\ Keywords \\ Sub-Fractional Brownian Motion, Fractional Brownian Motion, Self-Similar Gaussian Processes, Strong Local Non-Determinism}

\section{Introduction}

The fractional Brownian motion (fBm for short) is the best known and most used process with long-dependence property for models in telecommunications, turbulence, image processing and finance. This process is first introduced by [1] and later studied by [2]. The self-similarity and stationarity of the increments are two main properties for which $\mathrm{fBm}$ enjoy success as a modeling tool. The $\mathrm{fBm}$ is the only continuous Gaussian process which is self-similar and has stationary increments; see [3]. Many authors have also proposed for using more general self-similar Gaussian processes and random fields as stochastic models; see e.g. [4]-[9]. Such applications have raised many interesting theoretical questions about self-similar Gaussian processes and fields in general. However, in contrast to the extensive studies on fractional Brownian motion, there has been little systematic investigation on other self-similar Gaussian processes until [10] fills the gap by developing systematic ways to study sample path properties of a class of self-similar Gaussian process, namely, the bifractional Brownian motion. Their main tools are the Lamperti transformation, which provides a powerful connection between self-similar processes and stationary processes; see [11], and the strong local non-determinism of Gaussian processes; see [12]. In particular, for any self-similar Gaussian processes $X=\{X(t), t \in \mathbb{R}\}$, the Lamperti transformation leads to a stochastic integal representation for $X$.

An extension of Bm which preserves many properties of the $\mathrm{fBm}$, but not the stationarity of the increments, is 
so called sub-fractional Brownian motion (sub-fBm, in short) introduced by [13]. The sub-fBm is another class of self-similar Gaussian process which has properties analogous to those of fBm; see [13]-[15]. Given a constant $H \in(0,1)$, the sub-fractional Brownian motion in $\mathbb{R}$ is a centered Gaussian process $X_{0}^{H}=\left\{X_{0}^{H}(t), t \in \mathbb{R}_{+}\right\}$with covariance function

$$
R^{H}(s, t):=R(s, t)=s^{2 H}+t^{2 H}-\frac{1}{2}\left[(s+t)^{2 H}+|s-t|^{2 H}\right]
$$

and $X_{0}^{H}(0)=0$.

Let $X_{1}^{H}, \cdots, X_{d}^{H}$ be independent copies of $X_{0}^{H}$. We define the Gaussian process $X^{H}=\left\{X^{H}(t), t \in \mathbb{R}_{+}\right\}$ with values in $\mathbb{R}^{d}$ by

$$
X^{H}(t)=\left(X_{1}^{H}(t), \cdots, X_{d}^{H}(t)\right), \quad \forall t \in \mathbb{R}_{+} .
$$

By (1), one can verify easily that $X^{H}$ is a self-similar process with index $H$, that is, for every constant $a>0$,

$$
\left\{X^{H}(a t), t \in \mathbb{R}_{+}\right\} \stackrel{d}{=}\left\{a^{H} X^{H}(t), t \in \mathbb{R}_{+}\right\},
$$

where $X \stackrel{d}{=} Y$ means that the two processes have the same finite dimensional distributions. Note that $X^{H}$ does not have stationary increments.

The strong local non-determinism is an important tool to study the sample path properties of self-similar Gaussian process, such as the small ball probability and Chung's law of the iterated logarithm. In this paper, we apply the Lamperti transformation to prove the strong local non-determinism of $X_{0}^{H}$. Throughout this paper, a specified positive and finite constant is denoted by $c_{i}$ which may depend on $H$.

\section{Strong Local Non-Determinism}

Theorem 1. For all constants $0<a<b, X_{0}^{H}$ is strongly locally $\varphi$-nondeterministic on $I=[a, b]$ with $\varphi(r)=r^{2 H}$. That is, there exist positive constants $c_{1}$ and $r_{0}$ such that for all $t \in I$ and all $0<r \leq \min \left\{t, r_{0}\right\}$,

$$
\operatorname{Var}\left(X_{0}^{H}(t)\left|X_{0}^{H}(s): s \in I, r \leq\right| s-t \mid \leq r_{0}\right) \geq c_{1} \varphi(r) .
$$

Proof. By Lamperti's transformation (see [11]), we consider the centered stationary Gaussian process $Y_{0}=\left\{Y_{0}(t), t \in \mathbb{R}\right\}$ defined by

$$
Y_{0}(t)=\mathrm{e}^{-H t} X_{0}^{H}\left(\mathrm{e}^{t}\right) \text {, for every } t \in \mathbb{R} .
$$

The covariance function $r(t):=\mathbb{E}\left(Y_{0}(0) Y_{0}(t)\right)$ is given by

$$
r(t)=\mathrm{e}^{-H t}\left\{1+\mathrm{e}^{2 H t}-\frac{1}{2}\left[\left(\mathrm{e}^{t}+1\right)^{2 H}+\left|\mathrm{e}^{t}-1\right|^{2 H}\right]\right\}=\mathrm{e}^{H t}\left\{\mathrm{e}^{-2 H t}+1-\frac{1}{2}\left[\left(1+\mathrm{e}^{-t}\right)^{2 H}+\left|1-\mathrm{e}^{-t}\right|^{2 H}\right]\right\},
$$

where $r(t)$ is an even function. By (6) and Taylor expansion, we verify that $r(t)=O\left(\mathrm{e}^{-\beta t}\right)$, as $t \rightarrow \infty$, where $\beta=\min \{H, 1-H\}$. It follows that $r(\cdot) \in L^{1}(\mathbb{R})$. Also, by using (6) and the Taylor expansion again, we also have

$$
r(t) \sim 2-\frac{1}{2}\left(2^{2 H}+|t|^{2 H}\right) \text { as } t \rightarrow 0 .
$$

Using Bochner's theorem, $Y_{0}$ has the following stochastic integral representation

$$
Y_{0}(t)=\int_{\mathbb{R}} \mathrm{e}^{\mathrm{i} \lambda t} W(\mathrm{~d} \lambda), \quad \forall t \in \mathbb{R},
$$

where $W$ is a complex Gaussian measure with control measure $\Delta$ whose Fourier transform is $r(\cdot)$. The measure $\Delta$ is called the spectral measure of $Y_{0}$. 
Since $r(\cdot) \in L^{1}(\mathbb{R})$, the spectral measure $\Delta$ of $Y_{0}$ has a continuous density function $f(\lambda)$ which can be represented as the inverse Fourier transform of $r(\cdot)$ :

$$
f(\lambda)=\frac{1}{\pi} \int_{0}^{\infty} r(t) \cos (t \lambda) \mathrm{d} t
$$

We would like to prove that $f$ has the following asymptotic property

$$
f(\lambda) \sim c_{2}|\lambda|^{-(1+2 H)} \text { as } \lambda \rightarrow \infty,
$$

where $c_{2}>0$ is an explicit constant depending only on $H$.

In the following we give a direct proof of (10) by using (9) and an Abelian argument similar to that in the proof of Theorem 1 of [16]. Without loss of generality, we assume that $\lambda>0$. Applying integration-by-parts to (9), we get

$$
f(\lambda)=-\frac{1}{\pi \lambda} \int_{0}^{\infty} r^{\prime}(t) \sin (t \lambda) \mathrm{d} t
$$

with

$$
r^{\prime}(t)=H \mathrm{e}^{H t}\left[1-\mathrm{e}^{-2 H t}+\frac{1}{2}\left(1+\mathrm{e}^{-t}\right)^{2 H-1}\left(\mathrm{e}^{-t}-1\right)-\frac{1}{2}\left|1-\mathrm{e}^{-t}\right|^{2 H-1}\left(1+\mathrm{e}^{-t}\right)\right] .
$$

We need to distinguish three cases: $2 H<1,2 H=1$ and $2 H>1$. In the first case, it can be verified from (12) that $r(t)=O\left(\mathrm{e}^{-\beta t}\right)$ as $t \rightarrow \infty$, hence $r^{\prime}(t) \in L^{1}(\mathbb{R})$, and

$$
r^{\prime}(t) \sim-H|t|^{2 H-1} \text { as } t \rightarrow 0 .
$$

We will also make use of the properties of higher order derivatives of $r(t)$. It is elementary to compute $r^{\prime \prime}(t)$ and verify that, when $2 H<1$, we have

$$
r^{\prime \prime}(t) \sim-H(2 H-1)|t|^{2 H-2} \text { as } t \rightarrow 0
$$

and $r^{\prime \prime}(t)=O\left(\mathrm{e}^{-\beta t}\right)$ as $t \rightarrow \infty$ which implies $r^{\prime \prime}(\cdot) \in L^{1}(\mathbb{R})$.

The behavior of the derivatives of $r(t)$ is simpler when $2 H=1$. (12) becomes

$$
r^{\prime}(t)=-\frac{1}{2} \mathrm{e}^{-\frac{t}{2}}
$$

and

$$
r^{\prime \prime}(t)=\frac{1}{4} \mathrm{e}^{-\frac{t}{2}}
$$

Hence, we have $r^{\prime}(0)=-\frac{1}{2}, r^{\prime \prime}(0)=\frac{1}{4}$, and both $r^{\prime}(\cdot)$ and $r^{\prime \prime}(\cdot)$ are in $L^{1}(\mathbb{R})$.

When $2 H>1$, it can be shown that (14) still holds, and $r^{\prime \prime}(t)=O\left(\mathrm{e}^{-\beta t}\right)$ as $t \rightarrow \infty$.

Now, we proceed to prove (10). First, we consider the case when $0<2 H<1$. By a change of variable, we can write

$$
f(\lambda)=-\frac{1}{\pi \lambda^{2}} \int_{0}^{\infty} r^{\prime}\left(\frac{t}{\lambda}\right) \sin t \mathrm{~d} t
$$

Hence,

$$
\frac{f(\lambda)}{-\left(\pi \lambda^{2}\right)^{-1} r^{\prime}(1 / \lambda)}=\int_{0}^{\infty} \frac{r^{\prime}(t / \lambda)}{r^{\prime}(1 / \lambda)} \sin t \mathrm{~d} t .
$$

Let $p \in(0, \infty)$ be a fixed constant. It follows from (13) and the dominated convergence theorem that

$$
\lim _{\lambda \rightarrow \infty} \int_{0}^{p} \frac{r^{\prime}(t / \lambda)}{r^{\prime}(1 / \lambda)} \sin t \mathrm{~d} t=\int_{0}^{p} t^{2 H-1} \sin t \mathrm{~d} t
$$


On the other hand, integration-by-parts yields

$$
\int_{p}^{\infty} r^{\prime}(1 / \lambda) \sin t \mathrm{~d} t=r^{\prime}(p / \lambda) \cos p+\frac{1}{\lambda} \int_{p}^{\infty} r^{\prime \prime}(t / \lambda) \cos t \mathrm{~d} t
$$

By Riemann-Lebesgue lemma,

$$
\frac{1}{\lambda} \int_{p}^{\infty} r^{\prime \prime}(t / \lambda) \cos t \mathrm{~d} t=\int_{p / \lambda}^{\infty} r^{\prime \prime}(x) \cos (\lambda x) \mathrm{d} x=\int_{-\infty}^{\infty} 1_{\{x \geq p / \lambda\}} r^{\prime \prime}(x) \cos (\lambda x) \mathrm{d} x \rightarrow 0 \text { as } \lambda \rightarrow \infty .
$$

Moreover, since $r^{\prime}\left(\frac{p}{\lambda}\right) \sim-H\left(\frac{p}{\lambda}\right)^{2 H-1}$ as $\lambda \rightarrow \infty$ by (13) and $\left(\frac{p}{\lambda}\right)^{2 H-1} \rightarrow \infty$ as $\lambda \rightarrow \infty$, we have $\left|r^{\prime}\left(\frac{p}{\lambda}\right)\right| \rightarrow \infty$ as $\lambda \rightarrow \infty$. It follows that

$$
\left|\frac{1}{\lambda} \int_{p}^{\infty} r^{\prime \prime}\left(\frac{t}{\lambda}\right) \cos t \mathrm{~d} t\right| \leq\left|r^{\prime}\left(\frac{p}{\lambda}\right)\right| \text { as } \lambda \rightarrow \infty
$$

Then for all $\lambda$ large enough, we derive

$$
\left|\int_{p}^{\infty} r^{\prime}(t / \lambda) \sin t \mathrm{~d} t\right| \leq\left|r^{\prime}(p / \lambda) \cos p\right|+\left|\frac{1}{\lambda} \int_{p}^{\infty} r^{\prime \prime}(t / \lambda) \cos t \mathrm{~d} t\right| \leq 2\left|r^{\prime}(p / \lambda)\right| .
$$

Hence, we have

$$
\limsup _{\lambda \rightarrow \infty}\left|\int_{p}^{\infty} r^{\prime}(t / \lambda) \sin t \mathrm{~d} t\right| \leq 2 p^{2 H-1} .
$$

Combining (18), (19), and (24), we have

$$
\begin{aligned}
& \lim _{\lambda \rightarrow \infty} \frac{f(\lambda)}{-\left(\pi \lambda^{2}\right)^{-1} r^{\prime}(1 / \lambda)}=\lim _{\lambda \rightarrow \infty} \int_{0}^{\infty} \frac{r^{\prime}(t / \lambda)}{r^{\prime}(1 / \lambda)} \sin t \mathrm{~d} t \\
& =\lim _{\lambda \rightarrow \infty} \int_{0}^{p} \frac{r^{\prime}(t / \lambda)}{r^{\prime}(1 / \lambda)} \sin t \mathrm{~d} t+\lim _{\lambda \rightarrow \infty} \int_{p}^{\infty} \frac{r^{\prime}(t / \lambda)}{r^{\prime}(1 / \lambda)} \sin t \mathrm{~d} t \\
& \rightarrow \int_{0}^{\infty} t^{2 H-1} \sin t \mathrm{~d} t \quad \text { as } p \rightarrow \infty .
\end{aligned}
$$

Then we see that, when $0<2 H<1$, (10) holds with $c_{2}=H \pi^{-1} \int_{0}^{\infty} t^{2 H-1} \sin t \mathrm{~d} t$.

Secondly, we consider the case $2 H=1$. Since $r^{\prime}(t)$ is continuous and $r^{\prime}(0)=-\frac{1}{2}$, (19) becomes

$$
\lim _{\lambda \rightarrow \infty} \int_{0}^{p} r^{\prime}(t / \lambda) \sin t \mathrm{~d} t=r^{\prime}(0) \int_{0}^{p} \sin t \mathrm{~d} t=r^{\prime}(0)(1-\cos p) .
$$

Using (20) and integration-by-parts again we derive

$$
\int_{p}^{\infty} r^{\prime}(t / \lambda) \sin t \mathrm{~d} t=r^{\prime}(p / \lambda) \cos p+\frac{1}{\lambda} \int_{p}^{\infty} r^{\prime \prime}(t / \lambda) \cos t \mathrm{~d} t
$$

It follows from the (27), (16) and Riemann-Lebesgue lemma that

$$
\lim _{\lambda \rightarrow \infty} \int_{p}^{\infty} r^{\prime}(t / \lambda) \sin t \mathrm{~d} t=r^{\prime}(0) \cos p .
$$

We see from the above and (17) that

$$
f(\lambda) \sim \frac{1}{2 \pi}|\lambda|^{-2} \text { as } \lambda \rightarrow \infty .
$$

This verifies that (10) holds when $2 H=1$. 
Finally we consider the case $1<2 H<2$. Note that (19) and (24) are not useful anymore and we need to modify the above argument. By using integration-by-parts to (11) we obtain

$$
f(\lambda)=-\frac{1}{\pi \lambda^{2}} \int_{0}^{\infty} r^{\prime \prime}(t) \cos (t \lambda) \mathrm{d} t
$$

Note that we have $-1<2 H-2<0$. Hence $r^{\prime \prime}(t)$ is integrable in the neighborhood of $t=0$. Consequently, the proof for this case is very similar to the case of $0<2 H<1$. From (30) and (14), we can verify that (10) holds as well and the constant $C_{2}$ is explicitly determined by $H$. Hence we have proved (10) in general.

It follows from (10) and Lemma 1 of [17] (see also [12] for more general results) that $Y_{0}=\left\{Y_{0}(t), t \in \mathbb{R}\right\}$ is strongly locally $\varphi$-nondeterministic on any interval $J=[-T, T]$ with $\varphi(r)=r^{2 H}$ in the following sense: There exist positive constants $\delta$ and $c_{3}$ such that for all $t \in[-T, T]$ and all $r \in(0,|t| \wedge \delta)$,

$$
\operatorname{Var}\left(Y_{0}(t)\left|Y_{0}(s): s \in J, r \leq\right| s-t \mid \leq \delta\right) \geq c_{3} \varphi(r) .
$$

Now we prove the strong local nondeterminism of $X_{0}^{H}$ on $I$. To this end, note that $X_{0}^{H}(t)=t^{H} Y_{0}(\log t)$ for all $t>0$. We choose $r_{0}=a \delta$. Then for all $s, t \in I$ with $r \leq|s-t| \leq r_{0}$ we have

$$
\frac{r}{b} \leq|\log s-\log t| \leq \delta \text {. }
$$

Hence, it follows from (31) and (32) that for all $t \in[a, b]$ and $r<r_{0}$,

$$
\begin{aligned}
& \operatorname{Var}\left(X_{0}^{H}(t)\left|X_{0}^{H}(s): s \in I, r \leq\right| s-t \mid \leq r_{0}\right) \\
& =\operatorname{Var}\left(t^{H} Y_{0}(\log t)\left|s^{H} Y_{0}(\log s): s \in I, r \leq\right| s-t \mid \leq r_{0}\right) \\
& =t^{2 H} \operatorname{Var}\left(Y_{0}(\log t)\left|Y_{0}(\log s): s \in I, r \leq\right| s-t \mid \leq r_{0}\right) \\
& \geq a^{2 H} \operatorname{Var}\left(Y_{0}(\log t)\left|Y_{0}(\log s): s \in I, \frac{r}{b} \leq\right| \log s-\log t \mid \leq \delta\right) \\
& \geq a^{2 H} c_{3}\left(\frac{r}{b}\right)^{2 H}=c_{1} r^{2 H}=c_{1} \varphi(r),
\end{aligned}
$$

where $c_{1}=c_{3}\left(\frac{a}{b}\right)^{2 H}$. This proves Theorem 1 .

\section{Funding}

Supported by NSFC (No. 11201068) and “The Fundamental Research Funds for the Central Universities” in UIBE (No. 14YQ07).

\section{References}

[1] Kolmogorov, A.N. (1940) Wienersche Spiralen und einige andere interessante Kurven im Hilbertschen Raum. C.R. (Doklady) Acad. Sci. URSS (N.S.), 26, 115-118.

[2] Mandelbrot, B. and van Ness, J.W. (1968) Fractional Brownian Motions, Fractional Noises and Applications. SIAM Review, 10, 422-437. http://dx.doi.org/10.1137/1010093

[3] Samorodnitsky, G. and Taqqu, M.S. (1994) Stable Non-Gaussian Random Processes. Stochastic Models with Infinite Variance. Stochastic Modeling. Chapman \& Hall, New York.

[4] Anh, V.V., Angulo, J.M. and Ruiz-Medina, M.D. (1999) Possible Long-Range Dependence in Fractional Random Fields. Journal of Statistical Planning and Inference, 80, 95-110. http://dx.doi.org/10.1016/S0378-3758(98)00244-4

[5] Benassi, A., Bertrand, P., Cohen, S. and Istas, J. (2000) Identification of the Hurst Index of a Step Fractional Brownian Motion. Statistical Inference for Stochastic Processes, 3, 101-111. http://dx.doi.org/10.1023/A:1009997729317

[6] Benson, D.A., Meerschaert, M.M. and Baeumer, B. (2006) Aquifer Operator-Scaling and the Effect on Solute Mixing and Dispersion. Water Resources Research, 42, W01415. http://dx.doi.org/10.1029/2004wr003755

[7] Bonami, A. and Estrade, A. (2003) Anisotropic Analysis of Some Gaussian Models. Journal of Fourier Analysis and Applications, 9, 215-236. http://dx.doi.org/10.1007/s00041-003-0012-2 
[8] Cheridito, P., Kawaguchi, H. and Maejima, M. (2003) Fractional Ornstein-Uhlenbeck Processes. Electronic Journal of Probability, 8, 14 p.

[9] Mannersalo, P. and Norros, I. (2002) A Most Probable Path Approach to Queueing Systems with General Gaussian Input. Computer Network, 40, 399-412. http://dx.doi.org/10.1016/S1389-1286(02)00302-X

[10] Tudor, C.A. and Xiao, Y. (2007) Sample Path Properties of Bifractional Brownian Motion. Bernoulli, 13, $1023-1052$. http://dx.doi.org/10.3150/07-BEJ6110

[11] Lamperti, J. (1962) Semi-Stable Stochastic Processes. Transactions of the American Mathematical Society, 104, 62-78. http://dx.doi.org/10.1090/S0002-9947-1962-0138128-7

[12] Xiao, Y. (2007) Strong Local Non-Determinism of Gaussian Random Fields and Its Applications. In: Lai, T.-L., Shao, Q.-M. and Qian, L., Eds., Asymptotic Theory in Probability and Statistics with Applications, Higher Education Press, Beijing, 136-176.

[13] Bojdecki, T., Gorostiza, L.G. and Talarczyk, A. (2004) Sub-Fractional Brownian Motion and Its Relation to Occupation Times. Statistics and Probability Letters, 69, 405-419. http://dx.doi.org/10.1016/j.spl.2004.06.035

[14] Dzhaparidze, K. and Van Zanten, H. (2004) A Series Expansion of Fractional Brownian Motion. Probability Theory and Related Fields, 103, 39-55. http://dx.doi.org/10.1007/s00440-003-0310-2

[15] Tudor, C. (2007) Some Properties of the Sub-Fractional Brownian Motion. Stochastics, 79, 431-448. http://dx.doi.org/10.1080/17442500601100331

[16] Pitman, E.J.G. (1968) On the Behavior of the Characteristic Function of a Probability Distribution in the Neighborhood of the Origin. Journal of the Australian Mathematical Society, 8 423-443. http://dx.doi.org/10.1017/S1446788700006121

[17] Cuzick, J. and Du Preez, J.P. (1982) Joint Continuity of Gaussian Local Times. Annals of Probability, 10, 810-817. http://dx.doi.org/10.1214/aop/1176993789 